doi: $10.2306 /$ scienceasia1513-1874.2014.40.313

\title{
On the minimum skew rank of graphs
}

\author{
Yanna Wang ${ }^{\mathrm{a}}$, Bo Zhou ${ }^{\mathrm{b}, *}$ \\ ${ }^{a}$ Public Courses Department, Hubei Industrial Polytechnic, Shiyan 442000, China \\ b School of Mathematical Sciences, South China Normal University, Guangzhou 510631, China
}

*Corresponding author, e-mail: zhoubo@ @scnu.edu.cn

Received 9 Jun 2013

Accepted 20 Mar 2014

\begin{abstract}
The minimum skew rank $\mathrm{mr}^{-}(\mathbb{F}, G)$ of a graph $G$ over a field $\mathbb{F}$ is the smallest possible rank among all skew symmetric matrices over $\mathbb{F}$ whose $(i, j)$ th entry (for $i \neq j$ ) is non-zero whenever $i j$ is an edge in $G$ and is zero otherwise. We characterize the graphs $G$ with cut vertices over an infinite field $\mathbb{F}$ such that $\mathrm{mr}^{-}(\mathbb{F}, G)=4$ determine the minimum skew rank of $k$-paths over a field $\mathbb{F}$, and show that $\mathrm{mr}^{-}(\mathbb{F}, G)=2 \beta(G)=\mathrm{MR}^{-}(\mathbb{F}, G)$ for a connected graph $G$ with no even cycles and a field $\mathbb{F}$ where $\beta(G)$ is the matching number of $G$, and $\mathrm{MR}^{-}(\mathbb{F}, G)$ is the largest possible rank among all skew symmetric matrices over $\mathbb{F}$.
\end{abstract}

KEYWORDS: skew-symmetric matrix, $k$-tree, $k$-path, zero forcing number, perfect matching

\section{INTRODUCTION}

We consider only simple graphs. Let $G$ be a graph with vertex set $V_{G}$ and edge set $E_{G}$. Let $\mathbb{F}$ be a field. An $n \times n$ matrix $A$ over $\mathbb{F}$ is skew-symmetric (respectively, symmetric) if $A^{\mathrm{T}}=-A$ (respectively, $A^{\mathrm{T}}=A$ ), where $A^{\mathrm{T}}$ denotes the transpose of $A$. For an $n \times n$ symmetric or skew-symmetric matrix $A$, the graph of $A$, denoted $G(A)$, is the graph with vertex set $\left\{v_{1}, v_{2}, \ldots, v_{n}\right\}$ and edge set $\left\{v_{i} v_{j}: a_{i j} \neq 0,1 \leqslant\right.$ $i<j \leqslant n\}$.

The minimum skew rank problem involves skew symmetric matrices and its study began recently ${ }^{1}$. If the characteristic of $\mathbb{F}$ is 2 , then a skew-symmetric matrix over $\mathbb{F}$ is also symmetric. Thus it is assumed throughout this paper that the characteristic of $\mathbb{F}$ is not 2.

For a field $\mathbb{F}$ and a graph $G$, let $S^{-}(\mathbb{F}, G)=$ $\left\{A \in \mathbb{F}^{n \times n}: A^{\mathrm{T}}=-A, G(A)=G\right\}$ be the set of skew-symmetric matrices over $\mathbb{F}$ described by $G$. The minimum skew rank of $G$ over $\mathbb{F}$, denoted by $\mathrm{mr}^{-}(\mathbb{F}, G)$ is defined as the minimum rank of matrices in $S^{-}(\mathbb{F}, G)$, and the corresponding maximum skew nullity of $G$, denoted by $M^{-}(\mathbb{F}, G)$, is defined as the maximum nullity of matrices in $S^{-}(\mathbb{F}, G)$. Obviously, $\operatorname{mr}^{-}(\mathbb{F}, G)+M^{-}(\mathbb{F}, G)=\left|V_{G}\right|$.

Let $K_{n}$ be the complete graph with $n$ vertices, and $K_{n_{1}, n_{2}, \ldots, n_{t}}$ the complete $t$-partite graph with $n_{i}$ vertices in the $i$ th partite sets for $i=1,2, \ldots, t$.

Note that the rank of a skew-symmetric matrix over $\mathbb{F}$ is always even. Thus $\mathrm{mr}^{-}(\mathbb{F}, G)$ is even for any field $\mathbb{F}$ and any graph $G$. As observed in Ref. 1, $\mathrm{mr}^{-}(\mathbb{F}, G)=0$ if and only if $G$ is an empty graph. If
$\mathbb{F}$ is infinite and $G$ is a connected graph with at least two vertices, then $\mathrm{mr}^{-}(\mathbb{F}, G)=2$ if and only if $G$ is a complete multipartite graph $K_{n_{1}, n_{2}, \ldots, n_{t}}$ for some $t \geqslant 2, n_{i} \geqslant 1$ for $i=1, \ldots, t$. An open question (Question 5.2) was posed in Ref. 1 to characterize the graphs $G$ such that $\mathrm{mr}^{-}(\mathbb{F}, G)=4$.

The $k$ th power $G^{k}$ of a graph $G$ is the graph whose vertex set is $V_{G}$, two distinct vertices being adjacent in $G^{k}$ if and only if their distance in $G$ is at most $k$. Let $P_{n}=v_{1} v_{2} \ldots v_{n}$ be the path on $n$ vertices. The minimum skew rank of the $k$ th power of a path over the real field $\mathbb{R}$ was determined in Ref. 2 .

The class of $k$-trees is defined recursively as follows ${ }^{3}$ : (i) The complete graph $K_{k+1}$ is a $k$-tree; (ii) a $k$-tree $G$ with $n+1$ vertices $(n \geqslant k+1)$ can be constructed from a $k$-tree $H$ on $n$ vertices by adding a vertex adjacent to all vertices of a $k$-clique of $H$. A $k$-path is a $k$-tree which is either $K_{k+1}$ or has exactly two vertices of degree $k$.

The maximum skew rank of a graph $G$ over a field $\mathbb{F}$, denoted by $\mathrm{MR}^{-}(\mathbb{F}, G)$, is defined as the maximum rank of matrices in $S^{-}(\mathbb{F}, G)$. Let $\beta(G)$ be the matching number of $G$. It was shown in Ref. 1 that

$$
\mathrm{mr}^{-}(\mathbb{F}, G)=2 \beta(G)=\mathrm{MR}^{-}(\mathbb{F}, G)
$$

for a tree $G$ and a field $\mathbb{F}$.

In this paper, we characterize the graphs $G$ with cut vertices over an infinite field $\mathbb{F}$ such that $\mathrm{mr}^{-}(\mathbb{F}, G)=4$, determine the minimum skew rank of $k$-paths over a field $\mathbb{F}$, from which we also deduce the minimum skew rank of the $k$ th power of a path 
over the real field $\mathbb{R}$, and show that (1) holds for a connected graph $G$ with no even cycles and a field $\mathbb{F}$.

\section{PRELIMINARIES}

Let $G$ be a graph. For $v \in V_{G}, G-v$ denotes the graph obtained from $G$ by deleting vertex $v$ (and all edges incident with $v$ ). For $X \subseteq V_{G}, G[X]$ denotes the subgraph of $G$ induced by vertices in $X$. We need the following lemmas established in Ref. 1.

Lemma 1 Let $G$ be a connected graph with at least two vertices and let $\mathbb{F}$ be an infinite field. Then $\mathrm{mr}^{-}(\mathbb{F}, G)=2$ if and only if $G$ is a complete multipartite graph.

For a field $\mathbb{F}$ and a graph $G$ with $v \in V_{G}$, let $r_{v}^{-}(\mathbb{F}, G)=\mathrm{mr}^{-}(\mathbb{F}, G)-\mathrm{mr}^{-}(\mathbb{F}, G-v)$. The union of graphs $G_{i}, i=1,2, \ldots, h$, denoted by $\cup_{i=1}^{h} G_{i}$, is the graph with vertex set $\cup_{i=1}^{h} V_{G_{i}}$ and edge set $\cup_{i=1}^{h} E_{G_{i}}$.

Lemma 2 Let $G$ be a graph with cut vertex $v$ and let $\mathbb{F}$ be a field where $G=\cup_{i=1}^{h} G_{i}$ and $\cap_{i=1}^{h} V_{G_{i}}=$ $\{v\}$. Then $\operatorname{mr}^{-}(\mathbb{F}, G)=\sum_{i=1}^{h} \mathrm{mr}^{-}\left(\mathbb{F}, G_{i}-v\right)+$ $\min \left\{\sum_{i=1}^{h} r_{v}^{-}\left(\mathbb{F}, G_{i}\right), 2\right\}$.

Lemma 3 Let $G$ be a graph and let $\mathbb{F}$ be an infinite field. If $G=G_{1} \cup G_{2}$ then $\mathrm{mr}^{-}(\mathbb{F}, G) \leqslant$ $\mathrm{mr}^{-}\left(\mathbb{F}, G_{1}\right)+\mathrm{mr}^{-}\left(\mathbb{F}, G_{2}\right)$.

Let $G$ be a graph. A subset $Z \subseteq V_{G}$ defines an initial colouring by colouring all vertices in $Z$ black and all the vertices outside $Z$ white. The colour change rule says: if a black vertex $u$ has exactly one white neighbour $v$, then change the colour of $v$ to black. In this case we write $u \rightarrow v$. The derived set of an initial colouring $Z$ is the set of vertices coloured black until no more changes are possible. A zero forcing set is a subset $Z \subseteq V_{G}$ such that the derived set of $Z$ is $V_{G}$. The zero forcing number of $G$, denoted by $Z(G)$, is the minimum size of a zero forcing set of $G$.

Lemma 4 Let $G$ be a graph and $\mathbb{F}$ a field. Then $M^{-}(\mathbb{F}, G) \leqslant Z(G)$.

Lemma 5 Let $G$ be a graph and $\mathbb{F}$ a field. Then $\mathrm{MR}^{-}(\mathbb{F}, G)=2 \beta(G)$.

Lemma 6 Let $G$ be a graph and $\mathbb{F}$ a field. If $H$ is an induced subgraph of $G, \mathrm{mr}^{-}(\mathbb{F}, H) \leqslant \mathrm{mr}^{-}(\mathbb{F}, G)$.

Lemma 7 Let $G$ be a graph with a unique perfect matching and $\mathbb{F}$ a field. Then $\mathrm{mr}^{-}(\mathbb{F}, G)=\left|V_{G}\right|$.

\section{RESULTS}

First we give a characterization of the graphs $G$ with cut vertices over an infinite field $\mathbb{F}$ such that $\mathrm{mr}^{-}(\mathbb{F}, G)=4$.

Theorem 1 Let $G$ be a graph with cut vertex $v$ and $\mathbb{F}$ an infinite field. Then $\mathrm{mr}^{-}(\mathbb{F}, G)=4$ if and only if one of the following conditions holds:

(i) $G=G_{1} \cup G_{2}$ and $V_{G_{1}} \cap V_{G_{2}}=\{v\}$, where $G_{1}, G_{2}$ are complete multipartite graphs such that $G_{1}-v$, $G_{2}-v$ are nonempty.

(ii) $G-v$ consists of a complete multipartite component and isolated vertices.

Proof: Suppose that $\mathrm{mr}^{-}(\mathbb{F}, G)=4$. Let $p$ be the number of complete multipartite components, and let $q$ be the number of isolated vertices in $G-v$. Let $m$ be the number of the remaining components. Note that the minimum skew rank of a graph that is neither a complete multipartite graph nor an empty graph is larger than 4.

Case 1. $q=0$. By Lemma 2, $4=\mathrm{mr}^{-}(\mathbb{F}, G) \geqslant$ $2 p+4 m$. If $m=1$, then $p=0$, a contradiction to the fact that $v$ is a cut vertex of $G$. Thus $m=0$, implying that $p=2$. Let $W_{1}$ and $W_{2}$ be the vertex sets of the two complete multipartite components of $G-v$ and let $G_{1}$ and $G_{2}$ be the subgraphs induced by $\{v\} \cup W_{1}$ and $\{v\} \cup W_{2}$, respectively. By Lemma 1 , $\mathrm{mr}^{-}\left(\mathbb{F}, G_{1}-v\right)=\mathrm{mr}^{-}\left(\mathbb{F}, G_{2}-v\right)=2$. Вy Lemma 2, $4=\mathrm{mr}^{-}(\mathbb{F}, G)=\mathrm{mr}^{-}\left(\mathbb{F}, G_{1}-v\right)+$ $\operatorname{mr}^{-}\left(\mathbb{F}, G_{2}-v\right)+\min \left\{r_{v}^{-}\left(\mathbb{F}, G_{1}\right)+r_{v}^{-}\left(\mathbb{F}, G_{2}\right), 2\right\}=$ $2+2+\min \left\{r_{v}^{-}\left(\mathbb{F}, G_{1}\right)+r_{v}^{-}\left(\mathbb{F}, G_{2}\right), 2\right\}$. Then $r_{v}^{-}\left(\mathbb{F}, G_{1}\right)=r_{v}^{-}\left(\mathbb{F}, G_{2}\right)=0$. Thus $\mathrm{mr}^{-}\left(\mathbb{F}, G_{1}\right)=$ $\mathrm{mr}^{-}\left(\mathbb{F}, G_{2}\right)=2$. By Lemma $1, G_{1}$ and $G_{2}$ are complete multipartite graphs, and then (i) follows.

Case 2. $q \neq 0$. Note that $r_{v}^{-}\left(\mathbb{F}, K_{2}\right)=2$. By Lemma 2, $4=\operatorname{mr}^{-}(\mathbb{F}, G) \geqslant 2 p+4 m+2$. Then $m=0$ and $p=1$, and thus (ii) follows.

Now suppose that (i) holds. Note that $G_{i}-$ $v$ is still a complete multipartite graph for $i=$ 1,2. By Lemma $1, \mathrm{mr}^{-}\left(\mathbb{F}, G_{1}\right)=\mathrm{mr}^{-}\left(\mathbb{F}, G_{2}\right)=$ $\mathrm{mr}^{-}\left(\mathbb{F}, G_{1}-v\right)=\mathrm{mr}^{-}\left(\mathbb{F}, G_{2}-v\right)=2$. Then $r_{v}^{-}\left(\mathbb{F}, G_{1}\right)+r_{v}^{-}\left(\mathbb{F}, G_{2}\right)=0$. Thus by Lemma 2 , $\mathrm{mr}^{-}(\mathbb{F}, G)=\mathrm{mr}^{-}\left(\mathbb{F}, G_{1}-v\right)+\mathrm{mr}^{-}\left(\mathbb{F}, G_{2}-v\right)+$ $\min \{0,2\}=4$.

Next suppose that (ii) holds. Let $W$ be the unique complete multipartite component, and let $a$ be the number of isolated vertices in $G-v$. By Lemma $1, \mathrm{mr}^{-}(\mathbb{F}, W)=2$. Note that $r_{v}^{-}\left(\mathbb{F}, K_{2}\right)=$ 2. Then by Lemma $2, \mathrm{mr}^{-}(\mathbb{F}, G)=\mathrm{mr}^{-}(\mathbb{F}, W)+a$. $\mathrm{mr}^{-}\left(\mathbb{F}, K_{1}\right)+2=2+0+2=4$.

Now we consider the minimum skew rank of $k$ paths. Note that a $k$-tree with at least $k+2$ vertices has 
at least two vertices of degree $k$ and any two vertices of degree $k$ are not adjacent. The following lemma follows directly from the definition of a $k$-path.

Lemma 8 Let $G$ be a k-path with at least $k+2$ vertices, and let $v$ be a vertex of $G$ with degree $k$. Then $G-v$ is also a k-path.

Let $G$ be a $k$-path with $n \geqslant k+2$ vertices. By Lemma 8, the vertices of $G$ may be labelled as follows: choose a vertex of degree $k$, label it as $v_{n}$, and label its unique neighbour of degree $k+1$ in $G$ as $v_{n-1}$. Then $v_{n-1}$ is a vertex of degree $k$ in the $k$ path $G-v_{n}$. Repeating the process above, we may label $n-k+1$ vertices of $G$ as $v_{n}, v_{n-1}, \ldots, v_{k+2}$. Obviously, $G-v_{n}-v_{n-1}-\cdots-v_{k+2}=K_{k+1}$ and it contains a vertex of degree $k$ in $G$, which is labelled as $v_{1}$, and the remaining vertices are labelled as $v_{2}$, $v_{3}, \ldots, v_{k+1}$ such that $v_{2}$ is the unique neighbour of $v_{1}$ with degree $k+1$ in $G$. Note that in our labelling, $v_{i}$ is not adjacent to $v_{j+1}, v_{j+2}, \ldots, v_{n}$ if $v_{i}$ is not adjacent to $v_{j}$ for $j \geqslant \max \{i+1, k+2\}$. Recall that a $k$-tree is a chordal graph. The above labelling is the 'perfect elimination' labelling inherent to chordal graphs ${ }^{4}$.

Theorem 2 Let $G$ be a k-path on $n$ vertices and $\mathbb{F}$ an infinite field. Then

$$
\mathrm{mr}^{-}(\mathbb{F}, G)= \begin{cases}n-k, & \text { if } n-k \text { is even }, \\ n-k+1, & \text { if } n-k \text { is odd } .\end{cases}
$$

Proof: First we show

$$
\mathrm{mr}^{-}(\mathbb{F}, G) \leqslant \begin{cases}n-k, & \text { if } n-k \text { is even, } \\ n-k+1, & \text { if } n-k \text { is odd }\end{cases}
$$

by induction on $n$. If $n=k+1$, then $G=K_{k+1}$, which is a complete multipartite graph, and thus by Lemma $1, \mathrm{mr}^{-}(\mathbb{F}, G)=2=n-k+1$. If $n=k+2$, then $G=K_{k+2}-e$ is also a complete multipartite graph, where $e \in E_{K_{k+2}}$, and thus by Lemma 1, $\mathrm{mr}^{-}(\mathbb{F}, G)=2=n-k$. Thus (2) is true for $n=k+1, k+2$. Suppose that $n \geqslant k+3$ and for a $k$-path $H$ on $m$ vertices with $k+1 \leqslant m \leqslant n-1$ we have

$$
\mathrm{mr}^{-}(\mathbb{F}, H) \leqslant \begin{cases}m-k, & \text { if } m-k \text { is even, } \\ m-k+1, & \text { if } m-k \text { is odd. }\end{cases}
$$

Let $G$ be a $k$-path on $n$ vertices. Let $G_{1}=$ $G\left[\left\{v_{1}, v_{2}, \ldots, v_{k+2}\right\}\right]$ and $G_{2}=G\left[\left\{v_{3}, v_{4}, \ldots, v_{n}\right\}\right]$. Then $G_{1}$ is a $k$-path on $k+2$ vertices, and $G_{2}$ is a $k$ path on $n-2$ vertices. Obviously, $\mathrm{mr}^{-}\left(\mathbb{F}, G_{1}\right)=2$, and by the induction hypothesis,

$$
\mathrm{mr}^{-}\left(\mathbb{F}, G_{2}\right) \leqslant \begin{cases}n-k-2, & \text { if } n-k-2 \text { is even, } \\ n-k-1, & \text { if } n-k-2 \text { is odd, }\end{cases}
$$

i.e.,

$$
\mathrm{mr}^{-}\left(\mathbb{F}, G_{2}\right) \leqslant \begin{cases}n-k-2, & \text { if } n-k \text { is even, } \\ n-k-1, & \text { if } n-k \text { is odd. }\end{cases}
$$

Note that $G=G_{1} \cup G_{2}$. By Lemma 3,

$$
\begin{aligned}
\mathrm{mr}^{-}(\mathbb{F}, G) & \leqslant \mathrm{mr}^{-}\left(\mathbb{F}, G_{1}\right)+\mathrm{mr}^{-}\left(\mathbb{F}, G_{2}\right) \\
& \leqslant 2+ \begin{cases}n-k-2, & \text { if } n-k \text { is even, } \\
n-k-1, & \text { if } n-k \text { is odd }\end{cases} \\
& = \begin{cases}n-k, & \text { if } n-k \text { is even, } \\
n-k+1, & \text { if } n-k \text { is odd. }\end{cases}
\end{aligned}
$$

This proves (2).

Next we show the reverse of (2) holds. Let $Z=$ $\left\{v_{1}, v_{2}, \ldots, v_{k}\right\}$. Colour all vertices in $Z$ black and all the vertices outside $Z$ white. We will show that $Z$ is a zero forcing set of $G$. Since all neighbours of $v_{1}$ that differ from $v_{k+1}$ are black, we have $v_{1} \rightarrow v_{k+1}$. Note that $v_{2}$ is adjacent to $v_{k+2}$ but not adjacent to $v_{k+3}, v_{k+4}, \ldots, v_{n}$. Since all neighbours of $v_{2}$ which differ from $v_{k+2}$ are black, we have $v_{2} \rightarrow$ $v_{k+2}$. Let $G_{1}=G\left[\left\{v_{1}, v_{2}, \ldots, v_{k+3}\right\}\right]$ and $G_{2}=$ $G\left[\left\{v_{1}, v_{2}, \ldots, v_{k+4}\right\}\right]$. If each neighbour of $v_{k+3}$ in $G_{1}$ is adjacent to $v_{k+4}$ in $G$, then $v_{k+4}$ is of degree $k+1$ in $G_{2}$, a contradiction. Thus there is a neighbour, say $w$, of $v_{k+3}$ in $G_{1}$ such that $w v_{k+4} \notin E_{G}$, and then $w v_{i} \notin E_{G}$ for $i \geqslant k+5$, implying that $w \rightarrow v_{k+3}$. Repeating the process above, we may finally colour all vertices of $G$ black. Thus $Z$ is a zero forcing set of $G$. By Lemma $4, M^{-}(\mathbb{F}, G) \leqslant Z(G) \leqslant k$, and then $\mathrm{mr}^{-}(\mathbb{F}, G)=n-M^{-}(\mathbb{F}, G) \geqslant n-k$. Note that the rank of a skew-symmetric matrix is even. It follows that

$$
\operatorname{mr}^{-}(\mathbb{F}, G) \geqslant \begin{cases}n-k, & \text { if } n-k \text { is even, } \\ n-k+1, & \text { if } n-k \text { is odd }\end{cases}
$$

as desired.

Obviously, $P_{n}^{k}$ is a complete graph if $k \geqslant$ $n$. Suppose that $k \leqslant n-1$. Obviously, $P_{n}^{k}\left[\left\{v_{1}, v_{2}, \ldots, v_{k+1}\right\}\right]=K_{k+1}$, and if $k \leqslant n-2$, then for $j=2,3, \ldots, n-k$, $P_{n}^{k}\left[\left\{v_{j}, v_{j+1}, \ldots, v_{k+j-1}\right\}\right]=K_{k}$, and $v_{k+j}$ is adjacent to $v_{j}, v_{j+1}, \ldots, v_{k+j-1}$. Thus $P_{n}^{k}$ is a $k$ path. Now by Lemma 1 and Theorem 2, we have the following result, which has been proved in Ref. 2 (when $\mathbb{F}$ is the real field $\mathbb{R}$ ). 
Corollary 1 Let $\mathbb{F}$ be an infinite field. Then

$\operatorname{mr}^{-}\left(\mathbb{F}, P_{n}^{k}\right)$
$\quad= \begin{cases}n-k, & 1 \leqslant k \leqslant n-1 \text { and } n-k \text { is even, } \\ n-k+1, & 1 \leqslant k \leqslant n-1 \text { and } n-k \text { is odd }, \\ 2, & k \geqslant n .\end{cases}$

From Ref. 1, (1) holds if $G$ is a tree (a connected graph with no cycles). Now we make a minor extension.

Theorem 3 Let $G$ be a connected graph with no even cycles and let $\mathbb{F}$ be a field. Then (1) holds.

Proof: By Lemma 5, $\mathrm{mr}^{-}(\mathbb{F}, G) \leqslant \mathrm{MR}^{-}(\mathbb{F}, G)=$ $2 \beta(G)$. Let $M$ be a maximum matching of $G$ and $\left\{v_{1}, \ldots, v_{k}\right\}$, the vertices in $M$. Then $M$ is a perfect matching of $H=G\left[\left\{v_{1}, \ldots, v_{k}\right\}\right]$. This perfect matching is unique. Otherwise, the graph induced by the vertices of the symmetric difference of two (different) perfect matchings of $H$ consists of even cycles, which is impossible because $G$ contains no even cycles. By Lemma 6 and Lemma $7, \mathrm{mr}^{-}(\mathbb{F}, G) \geqslant$ $\mathrm{mr}^{-}(\mathbb{F}, H)=2 \beta(G)$. Then the result follows.

Acknowledgements: This work was supported by the Specialized Research Fund for the Doctoral Programme of Higher Education of China (No. 20124407110002).

\section{REFERENCES}

1. Allion M, Bodine E, DeAlba LM, Debnath J, DeLoss L, Garnett C, Grout J, Hogben L, Im B, Kim H, Nair R, Pryporova O, Savage K, Shader B, Wehe AW (2010) Minimum rank of skew-symmetric matrices described by a graph. Lin Algebra Appl 432, 2457-72.

2. DeAlba LM, Kerzner E, Tucker S (2011) A note on the minimum skew rank of powers of paths. arXiv: 1107.2450 [math.CO].

3. Rose DJ (1974) On simple characterizations of $k$-trees. Discrete Math 7, 317-22.

4. Shier DR (1984) Some aspects of perfect elimination orderings in chordal graphs. Discrete Appl Math 7, 325-31. 